\title{
Recovery from apraxic deficits and its neural correlate
}

Mona Kusch $^{\mathrm{a}, \mathrm{b}}$, Claudia C. Schmidt ${ }^{\mathrm{b}}$, Lea Göden ${ }^{\mathrm{a}}$, Caroline Tscherpel ${ }^{\mathrm{a}, \mathrm{b}}$, Jutta Stahl ${ }^{\mathrm{c}}$, Jochen Saliger $^{\mathrm{d}}$, Hans Karbe ${ }^{\mathrm{d}}$, Gereon R. Fink ${ }^{\mathrm{a}, \mathrm{b}}$, Peter H. Weiss ${ }^{\mathrm{a}, \mathrm{b}}$

${ }^{a}$ Department of Neurology, University Hospital Cologne, 50924 Cologne, Germany

${ }^{b}$ Cognitive Neuroscience, Institute of Neuroscience \& Medicine (INM-3), Research Centre Jülich, 52425 Jülich, Germany

${ }^{c}$ Department of Psychology, University of Cologne, 50931 Cologne, Germany

${ }^{d}$ Neurological Rehabilitation Centre Godeshöhe, 53177 Bonn, Germany

* Corresponding author:

Claudia Schmidt, Cognitive Neuroscience, Institute of Neuroscience \& Medicine (INM-3), Research Centre Jülich, 52425 Jülich, Germany; phone: +49-2461-61-2084, fax: +49-246161-1518, email: c.schmidt@fz-juelich.de

Citation: Kusch M, Schmidt CC, Göden L, Tscherpel C, Stahl J, Saliger J, Karbe H, Fink GR, Weiss PH (2018). Recovery from apraxic deficits and its neural correlate. Restorative Neurology and Neuroscience, 36(6), 669-678. doi: 10.3233/RNN-180815

The final publication is available at IOS Press through http://dx.doi.org/10.3233/RNN$\underline{180815}$ 


\begin{abstract}
Background and Objective: Apraxia is a deficit of motor cognition leading to difficulties in actual tool use, imitation of gestures, and pantomiming object use. To date, little data exist regarding the recovery from apraxic deficits after stroke, and no statistical lesion mapping study investigated the neural correlate of recovery from apraxia. Accordingly, we here examined recovery from apraxic deficits, differential associations of apraxia task (imitation vs. pantomime) and effector (bucco-facial vs. limb apraxia) with recovery, and the underlying neural correlates.
\end{abstract}

Methods: We assessed apraxia in 39 patients with left hemisphere (LH) stroke both at admission and approximately 11 days later. Furthermore, we collected clinical imaging data to identify brain regions associated with recovery from apraxic deficits using voxel-based lesion-symptom mapping (VLSM).

Results: Between the two assessments, a significant recovery from apraxic deficits was observed with a tendency of enhanced recovery of limb compared to bucco-facial apraxia. VLSM analyses revealed that within the lesion pattern initially associated with apraxia, lesions of the left insula were associated with remission of apraxic deficits, whereas lesions to the (inferior) parietal lobe (IPL; supramarginal and angular gyrus) and the superior longitudinal fasciculus (SLF) were associated with persistent apraxic deficits. Conclusion: Data suggest that lesions affecting the core regions (and white matter) of the fronto-parietal praxis network cause more persistent apraxic deficits than lesions affecting other regions (here: the left insula) that also contribute to motor cognition and apraxic deficits.

Keywords: stroke, neurorehabilitation, aphasia, VLSM, insular cortex 


\section{Introduction}

The term apraxia refers to the inability to perform purposeful movements leading to difficulties in tool use, imitation of gestures, and pantomiming object use. Apraxia is a common consequence of left hemispheric (LH) stroke and is a deficit of motor cognition that cannot be explained solely by primary sensory or motor deficits, disturbed communication, or lack of motivation. Apraxia can manifest itself with the upper limbs, i.e., limb apraxia, or the face and mouth, i.e., bucco-facial apraxia. Several studies provide evidence for the clinical relevance of apraxia and its massive impact on care-giver dependency after discharge from the hospital or rehabilitation center (Dovern, Fink, \& Weiss, 2012).

Despite the importance of apraxia for the individual's prognosis concerning rehabilitation, the principles and course of recovery from apraxia are far from being understood. Investigating the recovery from limb and oral apraxia in $26 \mathrm{LH}$ stroke patients, Basso, Capitani, Della Sala, Laiacona, and Spinnler (1987) demonstrated a significant improvement in imitating gestures over a time course of eight months. A follow-up study conducted by the same group revealed no significant additional recovery in the following 1.5 years, highlighting the importance of the first few months post-stroke for the recovery from limb apraxia (Basso, Burgio, Paulin, \& Prandoni, 2000). On the other hand, Mimura, Fitzpatrick, and Albert (1996) provided evidence for long-term recovery from limb and bucco-facial apraxia in 15 LH stroke patients assessed around 4.5 months and 81.6 months post-stroke. In contrast, the group of $108 \mathrm{LH}$ stroke patients examined by Donkervoort, Dekker, and Deelman (2006) showed only little improvement of limb apraxia after eight and 20 weeks. Finally, $48 \%$ of the initially apraxic patients studied by Bickerton et al. (2012) had recovered at the follow-up after nine months (i.e., showed no further sign of deficits across all praxis tasks). Taken together, current evidence regarding the course of the recovery from apraxic deficits is far from being conclusive. 
Investigating the different apraxia tasks (i.e., imitation, pantomime, object use) in acute to subacute and chronic stroke patients, Stamenova, Black, and Roy (2011) showed that recovery could be detected in all tasks, albeit with a diverging recovery rate of acute to subacute versus chronic patients with respect to pantomiming: for deficits in pantomiming limb gestures, acute to subacute patients showed a faster recovery compared to chronic patients. Although the apraxic deficits were differentiated by different apraxia tasks, no direct comparison of the recovery courses for object use, imitation, and pantomime deficits was performed.

Thus, the studies mentioned above lack information on possible diverging recovery courses for the different critical characteristics of apraxia, as assessed by different apraxia tasks (i.e., imitation, pantomime, and object use), and for the different effectors affected by apraxia (i.e., limb and bucco-facial apraxia). Furthermore, most of the studies focused on the chronic stage of apraxia (> 3 months), although Basso et al. (2000) already drew attention to the importance of the early phase of rehabilitation for recovery from apraxia.

The current lack of knowledge regarding the course of recovery from apraxia is complemented by the dearth of data regarding the neural correlates of recovery from apraxic deficits. In LH stroke, limb apraxia has been associated with fronto-parietal lesions (Goldenberg, 2009; Haaland, Harrington, \& Knight, 2000), whereas bucco-facial apraxia has been related to lesions of the frontal and central operculum and the anterior part of the insular cortex (Raade, Rothi, \& Heilman, 1991; Tognola \& Vignolo, 1980). Neural substrates of defective pantomime of tool use in LH stroke include inferior frontal, temporal, and inferior parietal regions (Niessen, Fink, \& Weiss, 2014; Weiss et al., 2016), whereas imitation deficits have mainly been associated with areas within the parietal lobe including the intraparietal sulcus (Hoeren et al., 2014; Price et al., 2010). To the best of our knowledge, no study has thus far directly assessed the neural correlate of recovery from (different) apraxic deficits 
using, e.g., statistical lesion mapping. Basso et al. (1987) grouped patients according to their performance at the first examination and their amount of recovery (below/above median) and then visually compared the lesion patterns of these patient groups. They suggested that recovery from deficits in imitating gestures was associated with more frontal than parietal lesions. However, the lesion data of their follow-up study did not confirm this conclusion (Basso et al., 2000).

Accordingly, the current study aimed to further explore the recovery from apraxic deficits due to LH stroke. Note that the term 'recovery' is used in the current investigation to describe improved task performance in the apraxia tests between the two testing sessions, even if no (full) recovery of function was achieved (i.e., even if a patient still showed deficits in the apraxia test at the second assessment). For that purpose, we assessed apraxia in a group of LH stroke patients at admission to a standard rehabilitation program and about 11 days later. To investigate differential effects of task and effector, we evaluated different apraxia tasks (i.e., imitation, pantomime, and object use) as well as the effectors known to be differentially affected in patients suffering from apraxia, i.e., limb and face. To investigate the lesion correlates of recovery from apraxic deficits, we furthermore conducted voxel-based lesion-symptom mapping (VLSM) on the clinical imaging data of our $39 \mathrm{LH}$ stroke patients (Bates et al., 2003; Rorden, Karnath, \& Bonilha, 2007).

\section{Methods}

\subsection{Patients}

We consecutively recruited 54 patients with clinically diagnosed LH stroke from the Department of Neurology, University Hospital Cologne and the Neurological Rehabilitation Centre Godeshöhe, Bonn. We excluded 15 patients after enrolment for the following reasons: 
four due to additional right hemispheric lesions, three because of previous strokes, two patients with isolated midbrain lesions, two patients with additional neurological disorder (Parkinson's disease, amyloid angiopathy), two patients due to missing data at the second testing session, one patient with missing consent for obtaining his scans, and one patient who was older than 90 years.

Our final sample thus consisted of 39 patients with first-ever LH stroke, who fulfilled the inclusion criteria of being between 18 and 90 years old and having no psychiatric or other neurological disorder. The sample comprised of 22 men and 17 women with a mean age of 62.6 years $(S D=13.7$, range $=35-87$ years $)$. Twenty patients were recruited at the Department of Neurology, University Hospital Cologne and 19 patients at the Neurological Rehabilitation Centre Godeshöhe, Bonn. Thirty-four patients were right-handed (87.2\%), two were left-handed (5.1\%), and three ambidextrous (7.7\%), constituting a representative sample for handedness (Gutwinski et al., 2011). The mean time between stroke onset and first assessment was 20.3 days $(S D=21.9$, range $=1-102$ days $)$. Thirty-six patients had suffered from an ischemic, three from a hemorrhagic stroke. Lesions were confirmed by CT $(n=13)$ or MRI $(\mathrm{n}=26)$ scans.

All patients gave written informed consent before participating in the study. The study was carried out following the ethical principles of the World Medical Association (Declaration of Helsinki) and had been approved by the ethics committee of the Medical Faculty in Cologne.

\subsection{Procedure}

To measure recovery over the course of a rehabilitation program, patients performed a set of standardized neuropsychological tests once at admission (time post-admission: $M=4.9$ days, $S D=8.3$ days $)$ and a second time after a mean of 11.3 days $(S D=2.0$ days, range $=$ 
7-14 days). Between the two assessments, all patients received an individualized standard motor (i.e., physiotherapy and occupational therapy) and cognitive (i.e., speech and neuropsychological therapy) rehabilitation program, but did not receive any further specific rehabilitative treatment for apraxic deficits.

To quantify apraxia, the Cologne Apraxia Screening was applied (Kölner Apraxie Screening, KAS; Weiss et al., 2013). The KAS consists of four subtests assessing buccofacial and limb gestures with pantomime and imitation tasks (pantomiming the use of [buccofacial] objects related to the face, pantomiming the use of limb-related objects, imitating bucco-facial gestures, imitating limb gestures). The pantomime and imitation subtests each comprise five items for bucco-facial and limb gestures/objects, resulting in 20 items in total that contribute equally to the overall KAS score (maximum of four points for each of the 20 items, resulting in a maximum overall score of 80 points). For the pantomime subtests (bucco-facial- and limb-related objects alike), photos of different objects are presented whose handling the patient is instructed to pantomime (e.g., toothbrush, drinking glass, dice, scissors). For the evaluation of the pantomimes, one or two points (depending on the complexity of the movements) are given for the presence of predefined features of the pantomime (e.g., pantomiming the use of a toothbrush: the hand is almost closed to a fist; the hand is held laterally in front of the mouth; the mouth is slightly opened, and the teeth are shown; circling/pushing movements of the hand). Otherwise, no point is given when the feature is absent. For the imitation subtests, pictures showing a person who demonstrates different gestures are presented (e.g., sticking out the tongue, putting the thumb on the forehead). For the evaluation of the imitation (bucco-facial and limb gestures alike), four points are given for correct imitation on the first trial. Otherwise, the picture is shown again and two points are given for a successful second trial or no point for an erroneous second trial. The maximum score for each of the four subtests (consisting of five items) is 20 points. 
For the pantomime and imitation subscores, bucco-facial and limb gestures were pooled (maximum score: 40 points). For the bucco-facial and limb subscores, pantomime and imitation tasks were pooled (maximum score: 40 points). For further details of the KAS see also Dovern et al. (2012). Moreover, the De Renzi test for actual object use (De Renzi, Pieczuro, \& Vignolo, 1968) was performed. The praxis tasks were always performed with the left, i.e., ipsilesional hand.

For the assessment of aphasia, we administered the short form of the aphasia checklist (ACL-K; Kalbe, Reinhold, \& Kessler, 2002) consisting of the subtests: (i) reading aloud, (ii) auditory comprehension, (iii) verbal fluency, and (iv) a verbal communication rating by the examiner.

Furthermore, motor functions were assessed via the Action Research Arm Test (ARAT; Lyle, 1981) and the Medical Research Council (MRC; Medical Research Council of the United, 1978) paresis scale. The grip force of both hands was quantified using a vigorimeter (KLS Martin) and used to operationalize the degree of hemiparesis (of the right, contralesional hand).

\subsection{Statistical analyses}

\subsubsection{Behavioral data}

KAS performance was used to diagnose apraxia: based on the published cut-off criterion, LH stroke patients scoring less than 77 (of 80) points were classified as apraxic (Weiss et al., 2013). Based on the overall cut-off score of the KAS, separate cut-offs for the four apraxic domains (i.e., bucco-facial apraxia, limb apraxia, pantomime deficits, and imitation deficits) were defined: Patients scoring less than 39 points in a given domain were diagnosed as suffering from bucco-facial or limb apraxia as well as from pantomime or imitation deficits, respectively. As only 6 out of 39 patients exhibited object use deficits (i.e., 
scored below the cut-off of 30 in the De Renzi test for actual object use, $M=30.4, S D=3.9$, maximum score 32 ), we were unable to analyze recovery from object use deficits in the current study.

We quantified recovery from apraxia using a three-way repeated measures ANCOVA with time point (t1 and $\mathrm{t} 2$ ), task (pantomime and imitation), and effector (bucco-facial and limb gestures) as within-subject factors and the performance in the corresponding KAS subtests as the dependent variable. We included time post-stroke as a covariate to control for potential influences of the time elapsed between stroke and first assessment of apraxia.

Furthermore, we computed a recovery score (RS) for the overall KAS performance (i.e., $\mathrm{RS}_{\text {apraxia }}$ ) by subtracting the KAS performance score at the first testing session ( $\mathrm{t} 1$ ) from the KAS performance score at the second session ( $\mathrm{t} 2$ ). Therefore, a positive recovery score indicates a better performance at the second testing session (i.e., improvement of performance), whereas a negative score indicates a worse performance at the second compared to the first testing session (i.e., deterioration of performance). In the same vein, the performance in the ACL-K was used to assess recovery from aphasia ( $\left.\mathrm{RS}_{\text {aphasia }}\right)$. To quantify the recovery from hemiparesis in the affected, i.e., contralesional limb, grip force was used $\left(\mathrm{RS}_{\mathrm{paresis}}\right)$. We normalized grip force of the contralesional (i.e., right) hand by the grip force of the ipsilesional (i.e., left) hand to account for pre-existing individual differences in muscle strength.

To evaluate potential learning effects, we computed the correlation between the time elapsed between the two testing sessions and the recovery score for the overall KAS performance using Pearson correlation. The rationale for this analysis was that if recovery from apraxic deficits is mainly due to practice effects an even more pronounced recovery should appear when the time interval between the first and second assessment was short. Moreover, to verify that the accuracy of the KAS was sufficient to assess improvements 
between the first and second testing session, we calculated the standard error of measurement (SEM) for the KAS using the formula $S E M=S D \cdot \sqrt{1-r \alpha}$, with $S D$ being the standard deviation and $r \alpha$ the reliability of the test (Cronbach's alpha).

To assess the potential impact of the standard rehabilitation program on the recovery from apraxic deficits, we conducted correlation analyses between the recovery score for the overall KAS performance and the total amount of motor and cognitive therapy (in minutes), respectively, using Pearson correlation.

All statistical analyses were performed with the statistical software package SPSS 23 and the Type-I error level was set to .05 .

\subsubsection{Lesion data}

Lesion mapping was conducted using MRIcron software (www.mccauslandcenter.sc.edu/mricro/mricron/index.html). Lesions were manually drawn by an experimenter (CS) on axial slices of a T1-weighted template brain (ch2.nii.gz) in steps of 5mm. A second independent experimenter (CT) crosschecked all lesions and both evaluators had to agree upon lesion location and extent. CS and CT were blinded concerning the test performance of the patients to exclude any selection bias. Based on these lesion maps, different VLSM analyses (Bates et al., 2003; Rorden et al., 2007) were performed to associate brain regions with specific behavioral performance parameters at the voxel-level using the non-parametric mapping (NPM) program (distributed with MRIcron).

We conducted VLSM analyses with the initial overall KAS performance and the initial test performance in the four apraxic domains as the behavioral variables to find brain regions associated with (different) apraxic deficits. To reveal brain regions associated with the recovery from apraxic deficits, we performed a VLSM analysis with the recovery score for the overall KAS performance ( $\left.\mathrm{RS}_{\text {apraxia }}\right)$ as the behavioral variable. Finally, we looked for 
brain regions associated with persistent apraxic deficits by computing a VLSM analysis with the KAS performance at the second testing session.

We included only voxels that were damaged in at least $10 \%$ of the patients in these analyses. We calculated voxelwise $t$-statistics and controlled for multiple comparisons using the false discovery rate (FDR) correction (Kimberg, Coslett, \& Schwartz, 2007).

The VLSM maps were yoked to the Automatic Anatomical Labeling (AAL; TzourioMazoyer et al., 2002) and Johns Hopkins University white matter (JHU; Wakana, Jiang, Nagae-Poetscher, van Zijl, \& Mori, 2004) atlas templates provided with MRIcron to determine the brain regions comprising significant voxels.

\section{Results}

\subsection{Behavioral data}

Based on the overall KAS performance, 21 of our 39 patients (54\%) were found to be apraxic at the time of first assessment (for a lesion overlay of the patients initially suffering from apraxia see Figure 1B). Seventeen patients suffered from bucco-facial and limb apraxia. Four patients had limb apraxia only. Nineteen patients exhibited pantomime and imitation deficits. One patient exhibited an isolated pantomime and another one an isolated imitation deficit.

There was a significant correlation between the scores of the apraxia (i.e., KAS) and aphasia (i.e., ACL-K) tests $(r=.734, p<.001)$, indicating a strong association between the severity of apraxic and aphasic deficits in the current patients with LH stroke at the time of first assessment (t1). Regarding the (co-)occurrence of apraxic and aphasic deficits, apraxia without aphasia was rare $(n=3,8 \%)$, while the commonest pattern $(n=16,43 \%)$ was comorbid apraxia and aphasia. Thus, more than two-thirds $(n=16,70 \%)$ of all aphasic patients 
$(\mathrm{n}=23)$ were also apraxic. Conversely, $84 \%(\mathrm{n}=16)$ of all apraxic patients $(\mathrm{n}=19)$

additionally suffered from aphasia. Aphasia without apraxia was present in 19\% (n=7) of all patients. Eleven patients (30\%) did not show any apraxic or aphasic deficits. Since the ACLK score was not available for two patients, this analysis included 37 patients only.

With respect to motor functions, 25 patients exhibited paresis of the contralesional (i.e., right) $\operatorname{limb}(\mathrm{MRC}$ paresis scale: $M=3.4, S D=1.9$ ). Apraxia, aphasia, and motor scores for all LH stroke patients at the time of first (t1) and second assessment (t2) are listed in Table 1.

Looking at the recovery from apraxic deficits, there was a significant improvement in the KAS performance between the two testing sessions even after controlling for the effect of time post-stroke $\left[F(1,37)=11.789, p=.001, \eta p^{2}=.242\right]$. There was neither a significant main effect of task $[F(1,37)=0.281, p=.599]$ nor a significant interaction between time of assessment and task $[F(1,37)=0.117, p=.734]$. In contrast, there was a significant main effect of effector $\left[F(1,37)=4.180, p=.048, \eta p^{2}=.101\right]$ indicating that patients performed limb-related gestures worse than bucco-facial gestures, and a marginally significant interaction effect between time of assessment and effector $\left[F(1,37)=3.661, p=.063, \eta p^{2}=\right.$ .090] with a tendency of more pronounced recovery from limb compared to bucco-facial apraxia (see Figure 2). Note that the initial apraxic deficits for bucco-facial gestures were less pronounced compared to limb related gestures (see also Table 1). The correlation between recovery from bucco-facial and limb apraxia was significant $(r=.675, p<.001)$.

Notably, there was no significant correlation between time between the testing sessions and the overall recovery score for apraxic deficits $(r=.024, p=.885)$. Given a Cronbach's alpha of 0.968 and a standard deviation of 19.20, the standard error of measurement for the KAS overall test $\left(\mathrm{SEM}_{\mathrm{KAS}}\right)$ was 3.43. Sixteen subjects of the 21 patients 
initially suffering from apraxia exhibited a (positive) recovery score larger than the $\mathrm{SEM}_{\mathrm{KAS}}$.

Despite the significant improvement in overall KAS performance during the interval between first and second assessments, apraxic patients still performed worse than nonapraxic patients in the apraxia test (i.e., KAS) at the second testing session $[t(37)=3.703, p=$ $.001]$. Based on the overall KAS performance, 16 of the 21 initially apraxic patients (76\%) were still apraxic at the time of the second assessment (i.e., scored below the cut-off score of 77 points).

Concerning the standard rehabilitation program, on average patients received treatment for seven days between their first (t1) and second (t2) assessment, with 90 to 120 minutes of motor therapy (i.e., physiotherapy and occupational therapy) per day and about 45 minutes of cognitive therapy (i.e., speech and neuropsychological therapy) per day. Correlation analyses showed that the amount of motor and cognitive therapy time was not significantly related to the apraxia recovery score $(r=.213, p=.200$ and $r=.210, p=.205$, respectively).

Regarding the association of recovery from apraxic deficits with i) the initial apraxia severity, ii) the initial aphasia severity (as operationalized by the ACL-K score), and iii) the degree of contralesional paresis, there were significant negative correlations between the initial apraxia and aphasia severity and the recovery score for apraxia $(r=-.593, p<.001$ and $r=-.702, p<.001$, respectively), indicating that more severe apraxic and aphasic deficits at t1 were associated with better recovery from apraxia. In contrast, there was no significant correlation between the initial normalized grip force and $\mathrm{RS}_{\text {apraxia }}(r=-.295, p=.069)$.

Conversely, the recovery from aphasia was not significantly correlated with initial severity of apraxia $(r=-.043, p=.800)$, but showed a strong statistical tendency to be significantly related to initial aphasia severity $(r=-.320, p=.054)$.

Comparing the recovery from apraxia $\left(\mathrm{RS}_{\mathrm{apraxia}}\right)$ with the recovery from aphasia 
$\left(\mathrm{RS}_{\text {aphasia }}\right)$ and paresis $\left(\mathrm{RS}_{\text {paresis }}\right)$, there were no significant correlations between the respective recovery scores, albeit the association between the recovery scores for apraxic and aphasic deficits showed a statistical tendency towards significance (apraxia-aphasia: $r=.298, p=$ .073 ; apraxia-paresis: $r=.115, p=.484)$.

\subsection{Lesion data}

Figure 1A displays a lesion overlay image of all 39 patients with LH stroke. As revealed by VLSM, apraxic deficits (operationalized by the overall KAS score) at the first testing session were significantly associated with cortical lesions of the frontal, temporal and inferior parietal cortex, the insula, and subcortical lesions affecting white matter and the basal ganglia $(p<.01$, FDR corrected, see Figure 3A, upper panel). Splitting up the different apraxia sub-tasks, pantomime deficits were associated with more anterior lesions, whereas imitation deficits were linked to more posterior lesions including the parietal cortex $(p<.01$, FDR corrected, see Supplementary Figure S1A and B). Bucco-facial and limb apraxia were both associated with large fronto-temporo-parietal lesions $(p<.01$, FDR corrected, see Supplementary Figure S1C and D). Concerning the brain correlates of recovery from apraxic deficits, lesions to the insular cortex were related to improved task performance in the apraxia test, i.e., recovery from apraxic deficits $(p<.01$, FDR corrected; Figure 3B, medium panel). Lesions affecting the basal ganglia (putamen and pallidum), the (inferior) parietal lobe (IPL; supramarginal and angular gyrus), and the superior longitudinal fasciculus (SLF) were associated with persistent apraxic deficits at the second testing session $(p<.01$, FDR corrected; Figure 3C, lower panel). 


\section{Discussion}

The aims of the current study were (i) to assess recovery from apraxic deficits after first-ever LH stroke, (ii) to investigate potentially differential recovery courses of buccofacial and limb apraxia as well as of pantomime and imitation deficits, and (iii) to identify the neural correlates of recovery from apraxic deficits.

When re-assessing apraxia in 39 LH stroke patients after approximately 11 days, we found a significant amelioration of apraxic deficits. In line with previous research (Basso et al., 1987; Mimura et al., 1996; Stamenova et al., 2011), we were thus able to find evidence for recovery from apraxic deficits - even within the short time interval applied in our study. Unfortunately, to the best of our knowledge, there is no apraxia scale with parallel versions to minimize practice effects. Likewise, no parallel version of the KAS exists. Therefore, familiarity with the test material may have contributed to the recovery from apraxic deficits between the first and second assessments in the current study. Note, however, that the time between the two testing sessions was not significantly related to recovery from apraxic deficits in the current patient sample rendering it unlikely that the improved task performance at the second testing session was solely due to practice effects.

When comparing the course of recovery concerning different apraxia domains, there was no differential effect for the recovery from imitation and pantomime deficits. However, there was a statistical tendency for a faster recovery from limb compared to bucco-facial apraxia. The fact that the recovery from bucco-facial and limb apraxia was nevertheless significantly correlated implies that patients who recover from limb apraxia also recover from bucco-facial apraxia. Importantly, previous studies by Basso et al. (1987; 2000) investigating the association between recovery from limb and oral apraxia also showed a significant correlation between the recovery from these two types of apraxic deficits, although Mimura et al. (1996) found that association only in non-fluent aphasics. 
As only six of our patients exhibited deficits in actual tool use our conclusions are limited to imitation and pantomime deficits. Studies drawing upon larger samples of patients are needed to examine the recovery from object use deficits, given that actual tool use is particularly relevant in the clinical context and leads to severe impairments in everyday life. Consistent with previous studies (e.g., Weiss et al., 2016), the co-occurrence of apraxic and aphasic deficits was the most common constellation in the current patients with LH stroke. Accordingly, the recovery from apraxic deficits significantly correlated with both (initial) apraxia and aphasia severity. Likewise, and in good accordance with previous research, recovery from apraxia was not significantly related to recovery from aphasia or paresis (Basso et al., 1987; Mimura et al., 1996), suggesting that the recovery from apraxic and aphasic deficits (that are both associated with LH lesions) depends - at least in part - on different processes/mechanisms. However, further investigations with even larger patient samples and more elaborate deficit assessments are warranted to examine common and specific neural (and cognitive) mechanisms of recovery from apraxia and aphasia.

Notably, the standard rehabilitation program (including physiotherapy and occupational therapy as well as speech and neuropsychological therapy, but no specific apraxia treatment) did not significantly impact on the recovery from apraxic deficits in the current patient sample. These findings suggest that no significant transfer across motor/cognitive domains occurred and stress once more the importance of establishing specific apraxia training programs in stroke rehabilitation (Dovern et al., 2012). Therefore, the current data provide essential information on the course of recovery from apraxic deficits for both clinicians and intervention studies investigating potential apraxia therapies.

With respect to the neural substrates of apraxic deficits and their recovery, the current VLSM analyses revealed that lesions affecting the inferior parietal lobe (i.e., supramarginal 
and angular gyrus) and the superior longitudinal fasciculus (SLF) of the left hemisphere were related to persistent apraxic deficits. These results are consistent with previous studies (e.g., Price et al., 2010; Thiebaut de Schotten et al., 2014) showing that lesions affecting the left inferior parietal cortex and the underlying white matter tracts may lead to chronic deficits. In contrast, lesions to the insular cortex were associated with initial, but not (or to a lesser extent) with persistent apraxic deficits. This finding suggests that patients with lesions affecting the insular cortex have a greater potential for recovering from apraxic deficits compared to patients with more parietal lesions. Note that this (statistical) finding does not necessarily mean that recovered patients suffered from lesions confined to the insula. Instead, it implies that in patients whose apraxic deficits improve a relevant portion of their lesion is located in the insular cortex, a region that does not belong to the core praxis network (comprising the parietal and frontal cortices as well as the underlying white matter tracts). This could imply that impairments in cognitive functions associated with insular lesions (contributing to the initial apraxic deficits) recover faster than the core praxis functions associated with parietal and frontal lesions. Putative cognitive functions that are associated with insular lesions and which are likely to be relevant for praxis comprise bodily selfconsciousness (Tsakiris, Hesse, Boy, Haggard, \& Fink, 2007), representation of body parts (Corradi-Dell'Acqua, Tomasino, \& Fink, 2009), or deficit awareness (Berti et al., 2005; Karnath, Baier, \& Nägele, 2005; Kusch et al., 2018). Thus, our data suggest that patients recover better from their (initial) apraxic deficits when these are - at least in part - due to cognitive deficits associated with the insula than when their apraxic deficits are mainly due to core motor functions affected by lesions in the fronto-parietal praxis network.

The current findings based on statistical lesion analysis extend previous observations made by Basso et al. (1987) who performed descriptive lesion analysis. These authors stated that especially temporo-parietal and occipital lesions hamper the recovery from deficits in 
imitating limb gestures. Our data further suggest that initial apraxic deficits due to insular lesions recover better than apraxic deficits caused by lesions within the (fronto-) parietal praxis network. How this recovery is promoted - either by spontaneous recovery or compensation - cannot be answered by the current data. Thus, further research is warranted to investigate the neural mechanisms that mediate putative compensatory processes during recovery from apraxia.

\section{Conclusion}

In our sample of $39 \mathrm{LH}$ stroke patients, we observed a significant recovery from apraxic deficits, while patients received a standard rehabilitation program, but no specific apraxia therapy. Our results suggest that patients show a better recovery from apraxic deficits when their (initial) apraxic deficits are caused by a lesion (here: the left insula) outside the core regions of the fronto-parietal praxis network. 


\section{Acknowledgments}

We are very grateful to all the patients who participated in our study. We also thank all our colleagues in the University Hospital Cologne, Research Centre Jülich and the Rehabilitation Centre Godeshöhe for their support. GRF gratefully acknowledges support from the Marga and Walter Boll foundation. 


\section{References}

Basso, A., Burgio, F., Paulin, M., \& Prandoni, P. (2000). Long-term follow-up of ideomotor apraxia. Neuropsychological Rehabilitation, 10(1), 1-13. doi:10.1080/096020100389264

Basso, A., Capitani, E., Della Sala, S., Laiacona, M., \& Spinnler, H. (1987). Recovery from ideomotor apraxia - a study on acute stroke patients. Brain, 110, 747-760.

Bates, E., Wilson, S. M., Saygin, A. P., Dick, F., Sereno, M. I., Knight, R. T., \& Dronkers, N. F. (2003). Voxel-based lesion-symptom mapping. Nature Neuroscience, 1-3. doi:10.1038/nn1050

Berti, A., Bottini, G., Gandola, M., Pia, L., Smania, N., Stracciari, A., ... Paulesu, E. (2005). Shared cortical anatomy for motor awareness and motor control. Science, 309. 488-491.

Bickerton, W.-L., Riddoch, M. J., Samson, D., Balani, A. B., Mistry, B., \& Humphreys, G. W. (2012). Systematic assessment of apraxia and functional predictions from the Birmingham Cognitive Screen. Journal of Neurology, Neurosurgery, and Psychiatry, 83(5), 513-521. doi:10.1136/jnnp-2011-300968

Corradi-Dell'Acqua, C., Tomasino, B., \& Fink, G.R. (2009). What is the position of an arm relative to the body? Neural correlates of body schema and body structural description. The Journal of Neuroscience, 29(13), 4162-4171. doi:10.1523/JNEUROSCI.486108.2009

De Renzi, E., Pieczuro, A., \& Vignolo, L. A. (1968). Ideational apraxia: a quantitative study. Neuropsychologia, 6(1), 41-52. doi:10.1016/0028-3932(68)90037-7

Donkervoort, M., Dekker, J., \& Deelman, B. (2006). The course of apraxia and ADL functioning in left hemisphere stroke patients treated in rehabilitation centres and nursing homes. Clinical Rehabilitation, 20(12), 1085-1093. doi:10.1177/0269215506071257

Dovern, A., Fink, G. R., \& Weiss, P. H. (2012). Diagnosis and treatment of upper limb apraxia. Journal of Neurology, 259(7), 1269-1283. doi:10.1007/s00415-011-6336-y

Goldenberg, G. (2009). Apraxia and the parietal lobes. Neuropsychologia, 47(6), 1449-1459. doi:10.1016/j.neuropsychologia.2008.07.014 
Gutwinski, S., Löscher, A., Mahler, L., Kabitzer, J., Heinz, A., \& Bermpohl, F. (2011). Understanding left-handedness. Deutsches Ärzteblatt International, 180(50), 849-853. doi:10.3238/arztebl.2011.0849

Haaland, K. Y., Harrington, D. L., \& Knight, R. T. (2000). Neural representations of skilled movement. Brain, 123 ( Pt 11), 2306-2313.

Hoeren, M., Kümmerer, D., Bormann, T., Beume, L., Ludwig, V. M., Vry, M.-S., ... Weiller, C. (2014). Neural bases of imitation and pantomime in acute stroke patients: distinct streams for praxis. Brain, 137(10), 2796-2810. doi:10.1093/brain/awu203

Kalbe, E., Reinhold, N., \& Kessler, J. (2002). Kurze Aphasie-Check-Liste (ACL-K). UCBPharma GmbH Kerpen.

Karnath, H.-O., Baier, B., \& Nägele, T. (2005). Awareness of the functioning of one's own limbs mediated by the insular cortex? The Journal of Neuroscience, 25(31), 7134-7138. doi:10.1523/JNEUROSCI.1590-05.2005

Kimberg, D. Y., Coslett, H. B., \& Schwartz, M. F. (2007). Power in voxel-based lesionsymptom mapping. Journal of Cognitive Neuroscience, 19(7), 1067-1080. doi:10.1162/jocn.2007.19.7.1067

Kusch, M., Gillessen, S., Saliger, J., Karbe, H., Binder, E., Fink, G.R. ... Weiss, P.H. (2018). Reduced awareness for apraxic deficits in left hemisphere stroke. Neuropsychology, 32(4), 509-515. doi:10.1037/neu0000451

Lyle, R. C. (1981). A performance test for assessment of upper limb function in physical rehabilitation treatment and research. International Journal of Rehabilitation Research, $4(4), 483-492$.

Medical Research Council of the United. (1978). Aids to examination of the peripheral nervous system: memorandum No 45. Palo Alta, CA: Pedragon House.

Mimura, M., Fitzpatrick, P. M., \& Albert, M. L. (1996). Long-term recovery from ideomotor apraxia. Neuropsychiatry Neuropsychology and Behavioral Neurology, 9(2), 127-132. 
Niessen, E., Fink, G. R., \& Weiss, P. H. (2014). Apraxia, pantomime and the parietal cortex. NeuroImage. Clinical, 5, 42-52. doi:10.1016/j.nicl.2014.05.017

Price, C. J., Crinion, J.T., Leff, A. P., Richardson, F. M., Schofield, T. M., Prejawa, S., ... Seghier, M. L. (2010). Lesion sites that predict the ability to gesture how an object is used. Archives Italiennes de Biologie, 148(3), 243-258.

Raade, A. S., Rothi, L. J., \& Heilman, K. M. (1991). The relationship between buccofacial and limb apraxia. Brain and Cognition, 16(2), 130-146.

Rorden, C., Karnath, H.-O., \& Bonilha, L. (2007). Improving lesion-symptom mapping. Journal of Cognitive Neuroscience, 19(7), 1081-1088. doi:10.1162/jocn.2007.19.7.1081

Stamenova, V., Black, S. E., \& Roy, E. A. (2011). A model-based approach to long-term recovery of limb apraxia after stroke. Journal of Clinical and Experimental Neuropsychology, 33(9), 954-971. doi:10.1080/13803395.2011.578570

Thiebaut de Schotten, M., Tomaiuolo, F., Aiello, M., Merola, S., Silvetti, M., Lecce, F., ... Doricchi, F. (2014). Damage to white matter pathways in subacute and chronic spatial neglect: a group study and 2 single-case studies with complete virtual "in vivo" tractography dissection. Cerebral Cortex, 24, 691-706. doi: 10.1093/cercor/bhs351

Tognola, G., \& Vignolo, L. A. (1980). Brain-lesions associated with oral apraxia in stroke patients - a clinico-neuroradiological investigation with the CT scan. Neuropsychologia, 18(3), 257-272.

Tsakiris, M., Hesse, M.D., Boy, C., Haggard, P., \& Fink, G.R. (2007). Neural signatures of body ownership: a sensory network for bodily self-consciousness. Cerebral Cortex, 17, 2235-2244. doi:10.1093/cercor/bhl131

Tzourio-Mazoyer, N., Landeau, B., Papathanassiou, D., Crivello, F., Etard, O., Delcroix, N., ... Joliot, M. (2002). Automated anatomical labeling of activations in SPM using a macroscopic anatomical parcellation of the MNI MRI single-subject brain. NeuroImage, 15, 273-289. doi:10.1006/nimg.2001.0978 
Wakana, S., Jiang, H., Nagae-Poetscher, L. M., van Zijl, P. C. M., \& Mori, S. (2004). Fiber tract-based atlas of human white matter anatomy. Radiology, 230(1), 77-87. doi:10.1148/radiol.2301021640

Weiss, P. H., Kalbe, E., Kessler, J., Fink, G. R., Binder, E., Hesse, M. D., \& Scherer, A. (2013). Kölner Apraxie Screening. Hogrefe: Göttingen.

Weiss, P. H., Ubben, S. D., Kaesberg, S., Kalbe, E., Kessler, J., Liebig, T., \& Fink, G. R. (2016). Where language meets meaningful action: a combined behavior and lesion analysis of aphasia and apraxia. Brain Structure and Function, 221, 563-576. doi:10.1007/s00429-014-0925-3 
Table 1. Descriptive data of the apraxia, aphasia, and motor scores for all LH stroke patients $(n=39)$ at the time of first (t1) and second assessment (t2)

t1 $\mathbf{t 2}$

\begin{tabular}{|c|c|c|}
\hline KAS overall & $68.2(16.0)$ & $72.4(13.2)$ \\
\hline KAS subtest pantomime & $33.8(8.8)$ & $35.5(7.9)$ \\
\hline KAS subtest imitation & $34.4(8.5)$ & $36.8(6.2)$ \\
\hline KAS subtest bucco-facial & $35.7(7.7)$ & $37.1(6.7)$ \\
\hline KAS subtest limb & $32.5(9.4)$ & $35.3(8.0)$ \\
\hline $\mathbf{R S}_{\text {apraxia }}$ & \multicolumn{2}{|c|}{$4.2(6.6)$} \\
\hline ACL-K & $25.1(12.3)^{\mathrm{a}}$ & $27.9(11.7)^{\mathrm{a}}$ \\
\hline $\mathbf{R S}_{\text {aphasia }}$ & \multicolumn{2}{|c|}{$2.5(5.0)^{\mathrm{a}}$} \\
\hline Grip force $(R / L)$ & $0.5(0.4)$ & $0.6(0.4)$ \\
\hline $\mathbf{R S}_{\text {paresis }}$ & \multicolumn{2}{|c|}{$0.0(0.3)$} \\
\hline
\end{tabular}

Means and standard deviations are provided.

KAS $=$ Cologne Apraxia Screening, ACL-K $=$ Aphasia Check List - short version, $\mathrm{RS}=$ recovery score, Grip force $(\mathrm{R} / \mathrm{L})=$ grip force of the contralesional (i.e., right) hand divided by the grip force of the ipsilesional (i.e., left) hand.

${ }^{\mathrm{a}} \mathrm{n}=37$ 


\section{Figure captions}

Figure 1. Lesion overlay of all LH stroke patients ( $=39$; A; upper panel) as well as of the patients initially suffering from apraxia $(n=21$; B; lower panel).

The color bars represent the number of overlapping lesions. Slices with the MNI-zcoordinates from -12 to 48 are shown.

Figure 2. Influence of effector (bucco-facial vs. limb gestures) on recovery from apraxic deficits in all LH stroke patients $(\mathrm{n}=39)$. Error bars indicate standard deviation.

Figure 3. Lesions associated with initial and persistent apraxic deficits as well with recovery from apraxic deficits in all LH stroke patients $(\mathrm{n}=39)$ as revealed by VLSM (all $p<.01$, FDR corrected).

A. Lesions significantly associated with initial apraxic deficits (as operationalized by the overall KAS score at the time of first assessment, $\mathrm{t} 1$; upper panel).

B. Lesions significantly associated with recovery from apraxic deficits (as operationalized by the difference in the overall KAS scores between the two assessments, t2-t1, $\mathrm{RS}_{\text {apraxia }}$; medium panel).

C. Lesions significantly associated with persistent apraxic deficits (as operationalized by the overall KAS score at the time of second assessment, $\mathrm{t} 2$; lower panel).

The color bars represent the corresponding t-values for the VLSM analysis. Slices with the MNI-Z-coordinates from -12 to 48 are shown. 
Figures

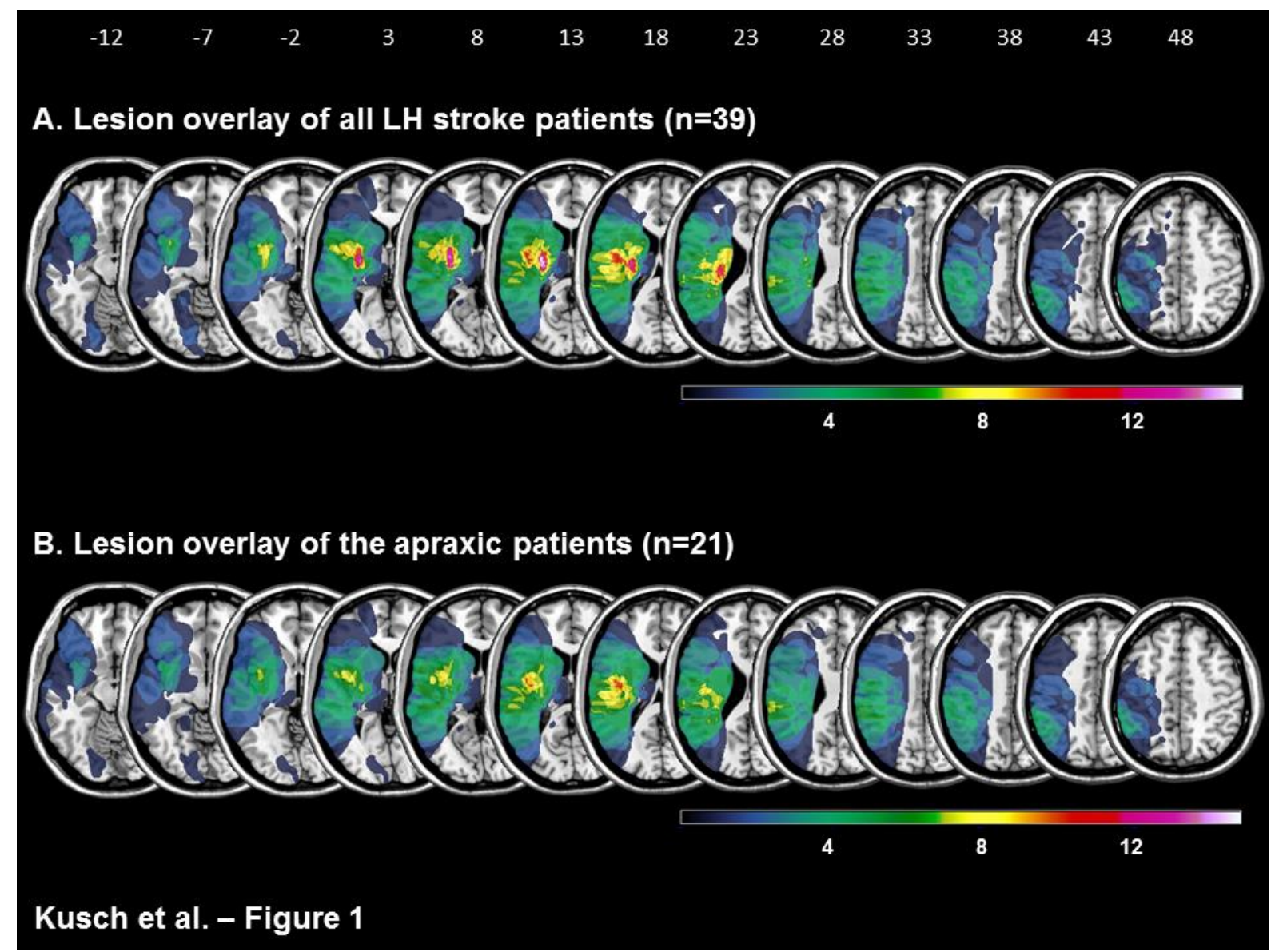




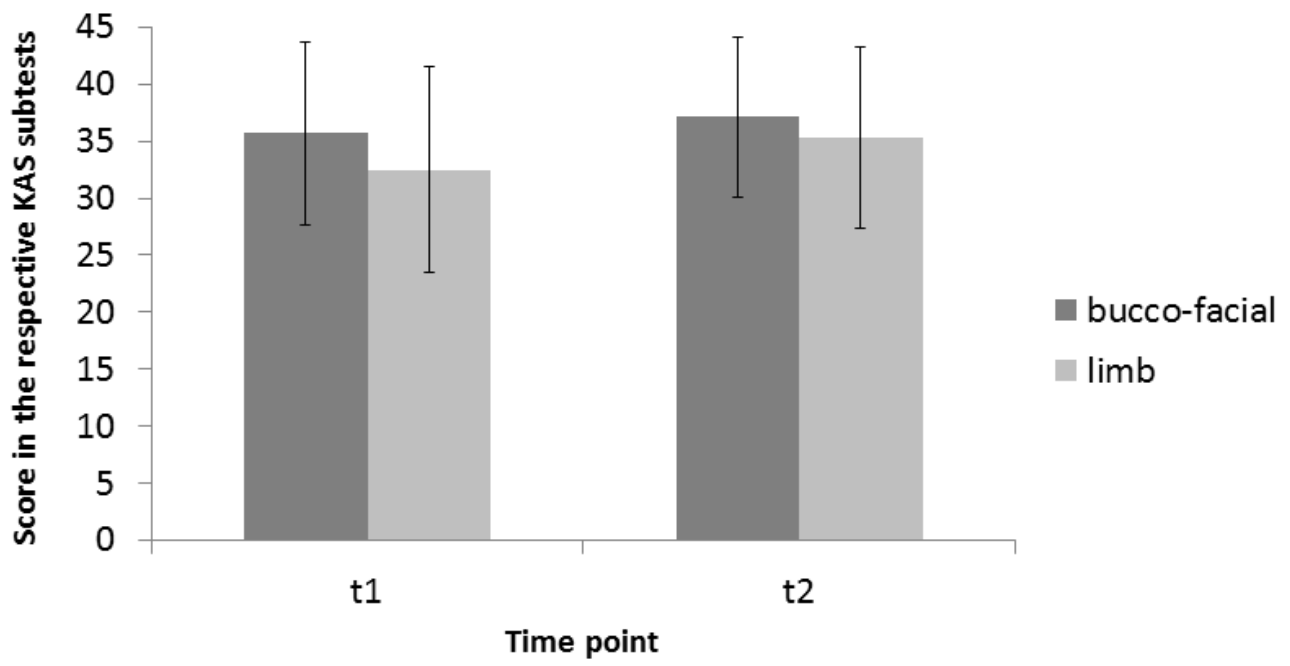

Kusch et al. - Figure 2 


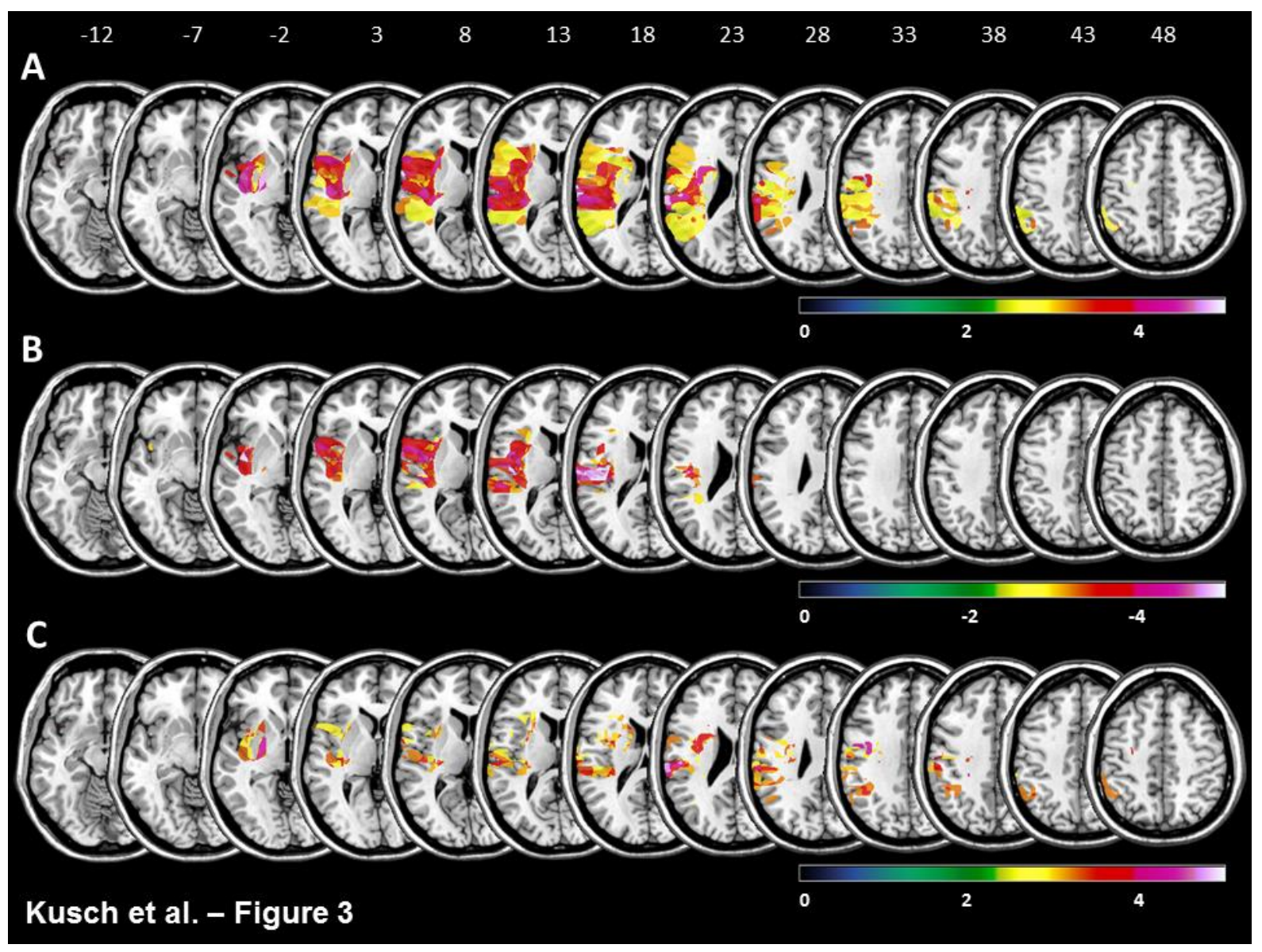




\section{Supplementary Material}



Supplementary Figure S1. Lesions associated with pantomime and imitation deficits as well as with bucco-facial and limb apraxia, separately, for all LH stroke patients $(\mathrm{n}=39)$ as revealed by VLSM (all $p<.01$, FDR corrected).

A. Lesions significantly associated with initial pantomime deficits (as operationalized by the KAS pantomime subscore at the time of first assessment, $\mathrm{t} 1$ ).

B. Lesions significantly associated with initial imitation deficits (as operationalized by the KAS imitation subscore at the time of first assessment, $\mathrm{t} 1$ ).

C. Lesions significantly associated with initial deficits in performing bucco-facial gestures (as operationalized by the KAS bucco-facial subscore at the time of first assessment, $\mathrm{t} 1$ ).

D. Lesions significantly associated with initial deficits in performing limb-related gestures (as operationalized by the KAS limb subscore at the time of first assessment, t1).

The color bar represents the corresponding t-values for the VLSM analysis. Slices with the MNI-z-coordinates from -12 to 48 are shown. 